J. Appl. Glycosci., 53, 205-213 (2006)

(C) 2006 The Japanese Society of Applied Glycoscience

Review

\title{
Current Understanding on Starch Granule Structures
}

\author{
(Received March 27, 2006; Accepted May 8, 2006) \\ Jay-lin Jane $e^{1, *}$ \\ ${ }^{1}$ Department of Food Science and Human Nutrition, Iowa State University \\ (Ames, Iowa 50011, USA)
}

\begin{abstract}
Starch is synthesized in semi-crystalline granular structures. Starches of different botanical origins possess different granular sizes, morphology, polymorphism and enzyme digestibility. These characteristics are related to the chemical structures of the amylopectin and amylose and how they are arranged in the starch granule. In this paper, structures and locations of amylose and amylopectin molecules in the granule are reviewed. The branch structures of amylopectin molecules and their relationship with the polymorphism, structures, and morphology of the starch granules are discussed. Internal structures of starch granules revealed by confocal laser-scattering microscopy and by using a surface-gelatinization method are compared and their effects on surface pinholes and serpentine channels of the starch granules are discussed.
\end{abstract}

Key words: starch granule, structure, amylose, amylopectin

Starch is the second largest biomass, next to cellulose, produced on earth and is the most available and economical commodity product. One major reason for starch being economically competitive is its semi-crystalline granular structure. Starch is produced in the granular form; therefore, it is easily isolated through wet milling. The starch can then be derivatized and modified with various chemical reagents, washed to remove by products and residual chemicals, and centrifuged to recover the modified starch that provides improved properties for various end uses and market needs.

Starch granules from different botanical sources have different characteristic shapes, sizes and morphology. ${ }^{1)}$ The sizes of starch granules vary from submicron to more than 100 microns in diameter. The shapes of starch granules include spherical, disk, oval, polygonal, dome-shape, elongated rod shape and compound starch. Most starch granules consist of two types of glucan, amylose and amylopectin. Amylopectin in the granule is present in the semi-crystalline structure, whereas amylose is amorphous. Starch granules possess different types of crystallinity, displaying A-, B- and C-type X-ray patterns, depending on their amylopectin branch chain-length. ${ }^{2}$ The A-type polymorphic starch has a monoclinic unit cell, and the Btype starch has a hexagonal unit cell., ${ }^{3,4)}$ The C-type polymorphic starch consists of a combination of the A-type and the B-type unit cells. Starches of different polymorphisms are known to display different enzyme digestibility. $^{5-9)}$ The A-type polymorphic starch is easily digestible, but the B-type and some C-type starches are very resistant to enzyme hydrolysis. The aim of this paper is to review some of the recent advances on the understanding of the

* Corresponding author (Tel.+1-515-294-9872, Fax.+1-515-2948181, E-mail: jjane@iastate.edu)

Abbreviations: DAP, days after pollination; DP, degree of polymerization; CLSM, confocal laser-scattering microscopy; HPAECENZ-PAD, high-performance anion-exchange chromatography equipped with an on-line enzyme reactor and a pulse amperometric detector. structure of starch granules and its impact on starch properties.

\section{Structure and location of amylose in the starch gran-} ule.

The structure of amylose has been extensively analyzed and is shown to have multiple branches; the number of branches of amylose molecules depends on the molecular size and botanical source of the starch. ${ }^{10,11)}$ Cereal starches have amylose of smaller molecular sizes than tuber and root starches. ${ }^{12,13)}$

\section{Locations of amylose versus amylopectin.}

Relative locations of amylose and amylopectin were a matter of debate among scientists for decades. On the basis of different properties of starch granules isolated from different botanical sources, including amylose leaching, iodine vapor complexing with amylose, amylose formation of the V-complex, and DMSO solubilization of starch granules, amylose is proposed of being separated from amylopectin in normal maize starch and being interspersed among amylopectin in potato starch. ${ }^{14)}$ Because amylose was known as linear molecules not being branched by starch branching enzymes during biosynthesis, it was hypothesized that amylose was synthesized in bundles, which was isolated from amylopectin and, thus, was not accessible to the starch branching enzymes. During the process of high-temperature/ethanol treatments to convert native starch granules into granular cold-watersoluble starch, normal starch granules preserved the granule integrity, but waxy starch granules dispersed. These phenomena are interpreted on the principle that amylose and amylopectin molecules in the normal starch granules are interspersed and intertwined with each other. Therefore, amylose holds the amylopectin molecules together during the thermal transition and, consequently, preserves the integrity of the starch granules. ${ }^{15)}$

Experiments have been designed to reveal whether amylose is dispersed among amylopectin or is separated from amylopectin and present in bundles. Selected cross- 
linking reagents of different chain lengths (between 4 and $7.5 \AA)$, including epichlorohydrin, adipic-acetic anhydride and phosphorus oxychloride, are applied and react with intact starch granules to cross-link molecules that are adjacent to each other. ${ }^{16)}$ Results of the cross-linking studies using normal maize and normal potato starches as the substrates and varying concentrations of cross-linking reagents show that amylose molecules are cross-linked with amylopectin and eluted with amylopectin at the void volume of gel permeation chromatograms. The blue value of the amylopectin peak increases with the increase in the reaction time, indicating that amylose is cross-linked onto amylopectin (Fig. 1).

When the concentration of the cross-linking reagent increases, adjacent amylopectin molecules are increasingly cross-linked and become insoluble. Amylose molecules extracted from the cross-linked starch that has a low to moderate level cross-linking, show no sign of increase in molecular weight, which suggests that no amylose molecules are cross-linked. In contrast, the molecular weight of amylose decreases after the starch has been subjected to the cross-linking reaction. This is attributed to the fact that amylose molecules of large molecular weights are preferentially cross-linked onto amylopectin and are eluted with the amylopectin at the void volume. ${ }^{16)}$ The amylose molecules isolated from phosphorus oxychloride crosslinked normal maize starch also show no phosphate diester cross-linkages as revealed by ${ }^{31} \mathrm{P}-\mathrm{nmr}$ spectrometry. The amylose molecules carry, however, exclusively phosphate monoester derivatives. ${ }^{17)}$ These results indicate that amylose molecules in the starch granules are not crosslinked by the bi-functional cross-linking reagents of chain length between 4 and $7.5 \AA$. The results support the concept that amylose molecules are not located in close vicinity of $4-7.5 \AA$ and are likely to be interspersed among amylopectin. The interspersed locations of amyloseamylopectin also agree with the fact that no retrograded amylose is found in native normal starch.

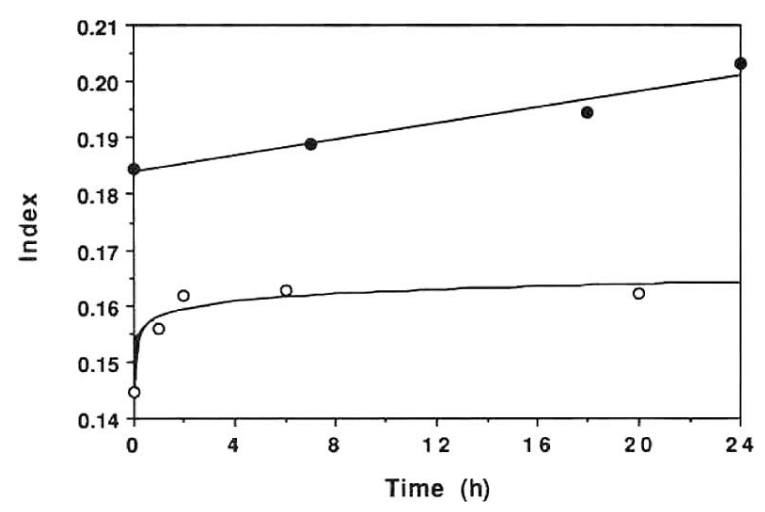

Fig. 1. Indices of amylose attachment to amylopectin in cornstarch $(\bigcirc)$ cross-linked with $0.0067 \%$ epichlorohydrin $(\mathrm{ECH} / \mathrm{starch}=\mathrm{w} / \mathrm{w})$ at $\mathrm{pH} 12.2$ in the presence of $2 \mathrm{M}$ sodium sulfate, and potato starch $(-)$ cross-linked with $0.0067 \% \mathrm{ECH}$ at $\mathrm{pH} 9.5$. The indices are plotted against the duration of the cross-linking reaction. Amylose and amylopectin were separated by a Sepharose CL-2B column $(2.5 \mathrm{~cm}$ i.d. $\times 80 \mathrm{~cm})$. Reprinted with permission from Ref. 16).

\section{Amylose is amorphous and oriented side by side with amylopectin in the starch granule.}

Starch granules are synthesized by apposition, ${ }^{18)}$ and the starch chains are elongated at the periphery of the starch granule ${ }^{19)}$ where the substrate is abundant. Combining all these facts, it suggests that amylose is synthesized side by side with amylopectin. Amylose is synthesized by granular-bound starch synthase, ${ }^{20)}$ which differs from amylopectin synthesized by soluble starch synthase. ${ }^{21)}$ It is proposed that branch-chains of amylopectin, elongated by the soluble starch synthase, have a greater tendency to interact with one another and form double helical crystalline structures. ${ }^{3)}$ Amylose is elongated in a possessive reaction pattern, ${ }^{22)}$ which does not have the same degree of mobility and the $\alpha 1-6$ branch linkage like the branch chains of amylopectin to interact with other chains to form double helices. Thus, amylose remains amorphous in the starch granule. Amylose molecules do carry a few branches. ${ }^{10,12)}$

\section{Amylose is concentrated at the periphery of the starch granule.}

The amylose content of starch increases with the maturation of the seeds and the size of starch granules..$^{23-26)}$ During the development of corn endosperms, the amylose content of starch increases from $9.2 \%$ on 12 days after pollination (DAP) to $24.2 \%$ on $30 \mathrm{DAP}^{27)}$ This increase in the amylose content of normal maize starch agrees with the increase in the activity of granular-bound starch synthase during the development of endosperm. ${ }^{28}$ When starch granules are treated with a saturated calcium chloride solution or a saturated lithium chloride solution, the starch granules are gelatinized on the surface by interacting with the cations that carry large charge density, such as $\mathrm{Ca}^{+2}$ and $\mathrm{Li}^{+1}$. The gelatinized starch at the periphery is then separated from the remaining granular starch, and both the inner and the outer fractions of the starch are subjected to starch structure analyses. The results obtained from the surface gelatinized starch show that the amylose content is greater at the periphery than that at the core of both potato ${ }^{24)}$ and normal maize ${ }^{25)}$ starch granules. These results are consistent with the increase in the amylose content as the starch granule grows larger. The results also agree with the report that granule-bound starchsynthase 1, the primary enzyme for amylose biosynthesis $^{29}{ }^{29}$ increases and maximizes at the late developmental stage of the storage organs. ${ }^{28}$ ) The granule-bound starch synthase is responsible for the biosynthesis of super-long branch chains of amylopectin. ${ }^{30,31}$

The increased concentration of amylose at the periphery of the normal starch granule is plausible to enhance the interaction and association between amylose and amylopectin. The tightly associated amylose and amylopectin network on the surface of the starch granules contributes to the ghost formation during cooking to prepare starch paste. The tightly associated amylose and amylopectin molecules at the periphery of starch granules are also less susceptible to enzyme hydrolysis and result in slower digestibility of uncooked normal starch granules. It is known that enzyme digestibility of granular starch is inversely related to the amylose content of the starch. For example, waxy rice and waxy maize starches are more easily digestible than normal rice and normal maize starch 
counterparts. Normal barley starch is more digestible than high-amylose barley starch, while both starches have similar amylopectin structures. ${ }^{9)}$

\section{Structure of amylopectin in the starch granule.}

Amylopectin is the major component of starch granules, varying between $100 \%$ in the waxy maize and waxy rice starch and about $60 \%$ in high-amylose maize starch. ${ }^{32)}$ Most normal starches consist of 20-30\% amylose. The molecular weight of amylopectin is larger than any other known natural or synthetic polymers. Therefore, there are no suitable reference compounds available for the calibration of the gel-permeation chromatogram to determine the molecular weight of amylopectin. Weight-average molecular weight of amylopectin determined by using highperformance size-exclusion chromatography equipped with multi-angle laser-light scattering and refractive index detectors show a range of $10^{8}$ to $10^{9} \mathrm{Da}$ for a wide variety of amylopectin molecules from different botanical sources, including cereal, roots, and tubers. ${ }^{9,33,34)}$ Glycogen from cyanobacteria displays a weight-average molecular weight of $2 \times 10^{7} \mathrm{Da}^{34)}$ The results reveal that the amylopectin of waxy starch possesses a larger weight-average molecular weight than the amylopectin of the normal starch counterpart. ${ }^{34)}$ This difference can be attributed to the fact that all of the ADP-glucose is used as the substrate for the biosynthesis of amylopectin in waxy starch, whereas ADP-glucose substrate is partitioning between the amylopectin and amylose for normal starch biosynthesis. Consequently, the molecular weights of normal starch amylopectin and high-amylose starch amylopectin are smaller than that of the waxy starch amylopectin.

Structures of amylopectin at different radial locations of starch granules.

Peripheral and inner core starches of potato ${ }^{24)}$ and normal maize starches ${ }^{25}$ are separated using the surface gelatinization method. Branch chains of the amylopectin molecules located at the periphery and at the inner part of the granule, determined using gelpermeation chromatography display different chain-length distributions. Amylopectin of peripheral starch (e.g., $8 \%$ surface gelatinized normal maize starch) consists of an average branch-chain length of DP 31.6 for the long B chains, which is shorter than that of the inner starch counterpart (e.g., DP 35.4 for the long $\mathrm{B}$ chains of the remaining starch granules after $65 \%$ starch removed by surface gelatinization). ${ }^{25)}$ These results agree with the fact that small starch granules consist of longer branch chains than the large starch granule counterparts. ${ }^{24,25)}$ Recent studies on the structures of normal maize amylopectin molecules during the development of maize kernels; using high-performance anion-exchange chromatography equipped with an on-line enzyme reactor and a pulse amperometric detector (HPAEC-ENZ-PAD), ${ }^{35)}$ showed that the average branch chains of amylopectin increased from DP 23.6 (10 DAP) to DP 26.9 (14 DAP) and then decreased to DP 24.9 on mature and dried starch (45 DAP). ${ }^{27)}$ These results are in agreement with the increase in expression of branching enzyme IIb, which is responsible for transferring short branch chains, during maize kernel development. ${ }^{36}$

Branch structures of amylopectin and the polymorphism of starch granules.

Amylopectin is responsible for the crystalline structure of starch granules. Native starch granules display 15-45\% crystallinity. ${ }^{14)}$ Amylopectin molecules have clustered branch-structures, ${ }^{37-41)}$ and the molecules consist of branch chains of different chain lengths. ${ }^{42}$ ) The average branch chain-length of the A-type polymorphic starch is shorter than the C- and the B-type starches. ${ }^{2}$ Average branch chain-length distributions of the A-, B- and C-type polymorphic starches determined using HPAEC-ENZ-PAD are shown in Fig. 2. The branch chain-length distributions of the different type starches show that the A-type polymorphic starch has a larger population of short branch chains, whereas the B-type polymorphic starch has fewer short branch chains but more long branch chains (B2, B3 and longer chains), which extend through two, three or more clusters. $^{42)}$ The C-type polymorphic starch has a mixture of the A-type and the B-type polymorphic structures, and thus has both the short and the long chains. The branch chain lengths of amylopectin and the polymorphism of the starch granules are in agreement with the results of in vitro studies using short amylose of different DP's as the substrate. ${ }^{43,44)}$ A super helical structure of amylopectin has been proposed by Oostergetel and van Bruggen, ${ }^{45}$ and blocklet structures of amylopectin have also been pro-

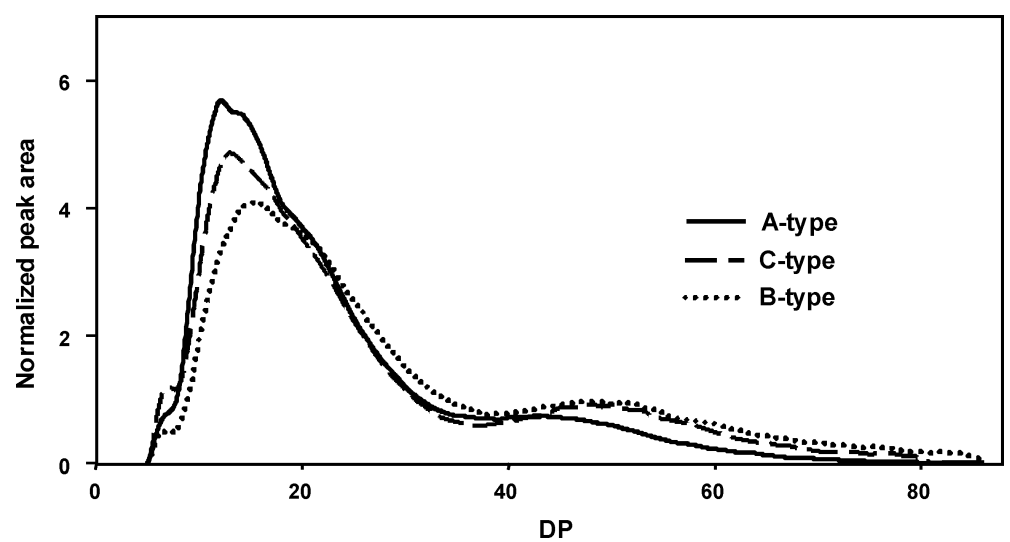

Fig. 2. Average branch chain-length distributions of amylopectins isolated from the starches of the A-, B- and the C-type polymorphism.

The branch chains of amylopectin were debranched by isoamylase and separated using high-performance anion-exchange chromatography equipped with an on-line enzyme reactor and a pulse amperometric detector (HPAEC-ENZ-PAD). ${ }^{35}$ A PA-100 column was used for the separation. 
posed. ${ }^{46,47)}$

Naegeli dextrins, prepared by exhaustive acid hydrolysis of granular starch, consist of highly crystalline structures. $^{48)}$ Fine structures of the Naegeli dextrin of waxy maize starch were analyzed using combinations of quantitative paper chromatography and selected enzymes, including saccharifying $\alpha$-amylase (from $B$. subtilis), $\beta$ amylase (from soybean), and pullulanase. ${ }^{49)}$ The results show that the Naegeli dextrin of waxy maize starch consists of $47 \%$ (molar ratio) linear chains (DP 8-17), 16\% branched chains that have the main unit-chain length of DP 12-16 carrying a glucosyl or maltosyl branch at the reducing end, $26 \%$ singly-branched chains carrying two unit chains with the peak chain-length of DP 14 (ranging between DP 7 and DP 20), and about 5\% chains having branches near the non-reducing end. ${ }^{49)}$ The fine structure of the Naegeli dextrin further supports the double helical structure of amylopectin branch chains in the granule. Starches of different polymorphisms produce Naegeli dextrins of different chain-length distribution patterns (Fig. 3). ${ }^{50)}$ The Naegeli dextrins of the A-type polymorphic starches display more singly branched chains, ${ }^{49,50)}$ whereas that of the B-type starches display fewer branched chains but more linear chains. ${ }^{50)}$ The branch chains of the Naegeli dextrins are likely results of the branch linkages that are protected and are not accessible to acid hydrolysis.

There are two possible mechanisms that either individually or jointly protects the branch linkages from being hydrolyzed by acid. One mechanism is that in the A-type starch, the branch linkages of the amylopectin are more scattered. This could be a result of large proportions of short $\mathrm{A}$ and $\mathrm{B} 1$ chains relative to the long-B chains. The
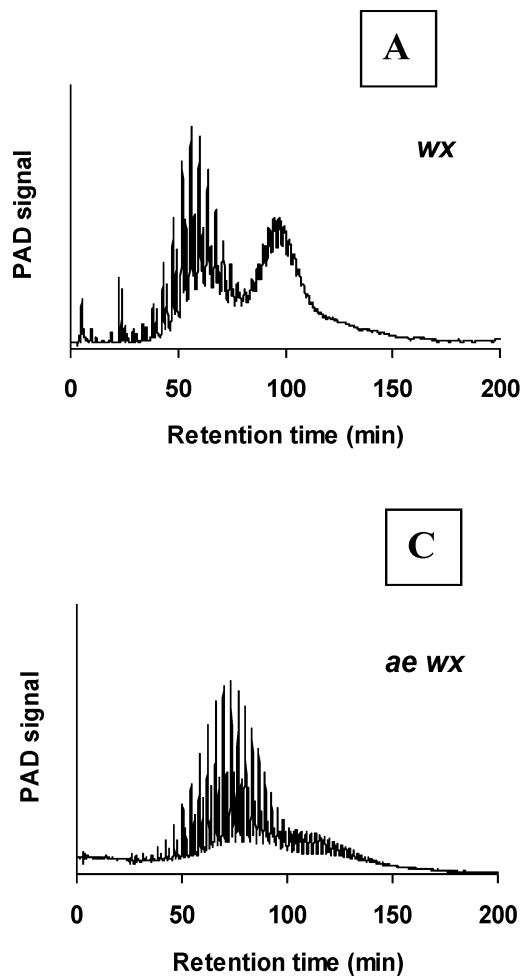

$\alpha$ 1-6 branch linkages have to be more scattered to accommodate the number of the branch linkages. This structure is supported by the results of small-angle X-ray and neutron scattering studies. ${ }^{14,51)}$ The distance of the crystalline region of the A-type polymorphic starch is similar to that of the B-type starch despite the significant differences in the branch chain-length of the two type starches. ${ }^{14,45,51)}$ When the branch linkages are scattered in the amorphous and the crystalline regions, those in the amorphous region are readily susceptible to the acid hydrolysis and produce linear chains. Whereas those located in the crystalline region are protected and remain as branched chains (Fig. 4).

This structure agrees with the results obtained by Hood and Mercier. ${ }^{52}$ Hood and Mercier show that hydroxypropyl derivatives of starch are concentrated in the region of $\alpha$ 1-6 branch linkages and a few hydroxypropyl groups are located at the non-reducing ends of short branch chains, the amorphous regions of amylopectin. ${ }^{52}$ The authors also find that $50 \%$ of the short chains of DP 15 contain no derivatives because they are located in the crystalline region. The short chains that are protected in the crystalline region and some short chains carrying the derivatives at the non-reducing end suggest that the branch linkages are scattered. For the B-type polymorphic starch, there are fewer short chains relative to the long Bchains. Thus, the branch linkages are possibly in a cluster and located in the amorphous region. Consequently, the branch linkages are readily hydrolyzed and produce mainly linear chains.

Another mechanism of the protection of the branch linkages in the A-type starch is that the A-type polymorphic starch has a monoclinic unit cell, which is closely packed. In contrast, the B-type polymorphic starch has a
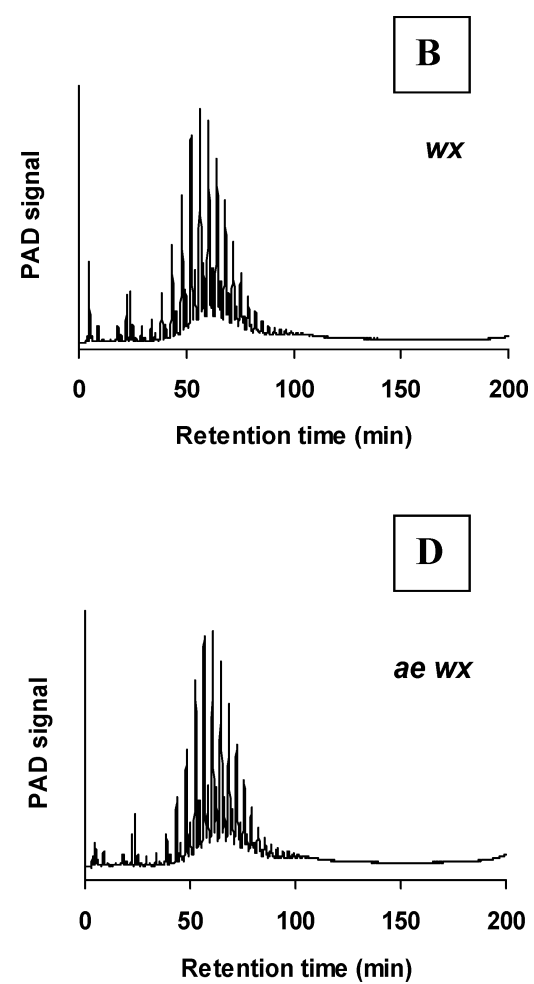

Fig. 3. Naegeli dextrins produced from the A- and the B-type polymorphic starches.

(A) Waxy maize starch (A-type polymorphic starch) Naegeli dextrin; (B) debranched waxy maize starch Naegeli dextrin; (C) ae waxy maize starch (B-type polymorphic starch) Naegeli dextrin; and (D) debranched ae waxy maize starch Naegeli dextrin. The dextrins were separated using the HPAEC-ENZ-PAD method described in Fig. 2. 


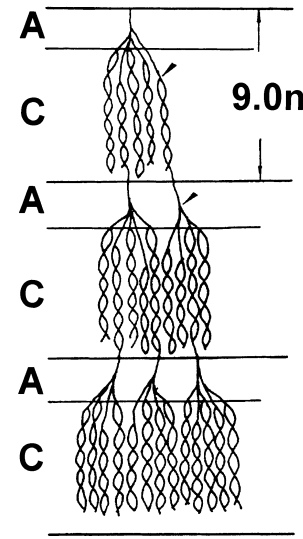

b

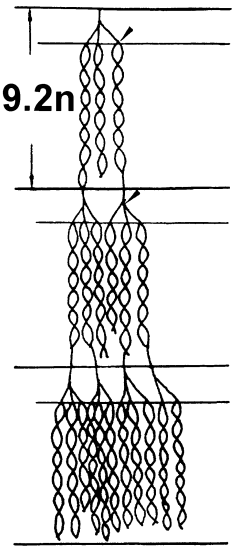

Fig. 4. Proposed models for branching patterns of (a) waxy maize starch (A-type polymorphic starch); and (b) potato starch (B-type polymorphic starch). "A" and "C" stand for the amorphous and crystalline regions, respectively; 9.0 and $9.2 \mathrm{~nm}$ are the repeating distances of waxy maize and potato starches, respectively. The arrows show the branch linkages in the two types of amylopectin molecules. Reprinted with permission from Ref. 50).

hexagonal unit cell, which is relatively loosely packed with an open channel of water in the unit cell. The closely packed A-type amylopectin can hinder the acid hydrolysis of its $\alpha$ 1-6 branch linkages and produce branched chains in the Naegeli dextrins. An example that supports this mechanism is that high-amylose 7 maize starch, which possesses the B-type polymorphism and consists of a large concentration of amylose, display more branched chains in the Naegeli dextrin. ${ }^{50)}$ In this case, the amylose molecules interspersed among amylopectin may protect the branch linkages of the amylopectin molecules from acid hydrolysis.

Relationships between the structure of amylopectin and the shape of starch granules.

Studies on the structures of amylopectin molecules isolated from the A- and B-granules of wheat, barley and triticale have shown that the amylopectin molecules of the disk-shaped, large A-granules and the spherical, small Bgranules have different structures. The amylopectin molecules of the A-granules consist of substantially more B2 chains that extend through two clusters and lesser $\mathrm{A}$ and B1 chains that remain in a single cluster than the amylopectin of the B-granules. ${ }^{53,54)}$ The amylopectin molecules that consist of more B2 chains extending through two clusters and carrying other B1 and A chains are likely to have cylindrical-shaped structures. ${ }^{54)}$ The cylindricalshaped molecules, in turn, can be aligned into a plateletstructure, which agrees with the parallel molecular arrangement in the disk-shaped starch granules revealed by optical map studies. ${ }^{38)}$ In the disk shaped starch granules, starch molecules are perpendicular to the flat surface of the disk-shaped granule. Consequently, there is an equatorial groove on the starch granule where starch molecules are less orderly packed and are more susceptible to enzyme and chemical attack. Whereas, the amylopectin of the B-granule starch consists of more A and B1 chains that remain in one cluster and fewer B2 chains. Amylopectin molecules consisting of more short chains and
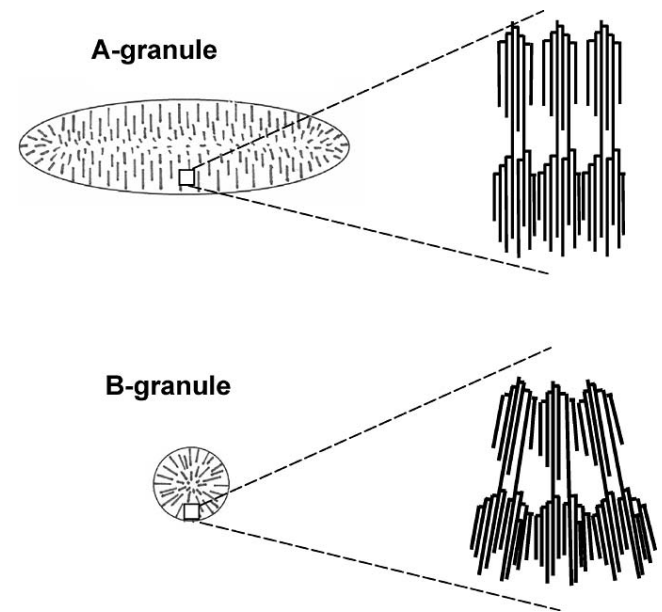

Fig. 5. Proposed granular and molecular structures of the A- (diskshaped) and B- (spherical) granules of wheat starch. The amylopectin branch-structure of the A- and B-granules are constructed on the basis of the molar ratios of short chains ( $A$ and $B_{1}$ chains) to long chains ( $B_{2}$ and longer chains) of amylopectin branch-chain numbers of wheat A- and Bgranule starches. Amylopectin molecules of the A-granule starch consist of more long chains but lesser short chains, which display a cylindrical shape and better align parallel into a disk-shaped A-granule. Amylopectin molecules of the B-granule starch consist of more short chains but lesser long chains, which display a cone shape. The coneshaped molecules fit in a spherical B-granule. Reprinted with permission from Ref. 54).

larger degrees of branching tend to have cone-shaped molecules and fit into a spherical starch granule. Models of the amylopectin structures and the starch granules are shown in Fig. 5. ${ }^{54}$

Parallel arranged cylindrical amylopectin molecules in the A-granule is expected to display larger crystallinity than the radial arranged cone-shaped amylopectin in the B-granule, which agrees with the results of crystallinity of waxy barley A-granule starch (36.6\%) and waxy barley B-granule starch (33.0\%). ${ }^{55}$ Normal barley A-granule starch, however, has less crystallinity than normal barley B-granule starch because the A-granule starch consists of more amylose $(28.1 \%)$ than the B-granule starch $(23.0 \%)$.

Internal structures of starch granules revealed by confocal laser-scattering microscopy.

Enzyme digestibility of starch granules varies substantially.,6,9) After $24 \mathrm{~h}$ hydrolysis using porcine pancreatic $\alpha$-amylase, waxy amaranth and waxy rice starch granules are completely hydrolyzed, and all starch granules that display the A-type polymorphism are hydrolyzed much faster than the B-type polymorphic starch granules. ${ }^{9)}$ It is well established that the crystalline structures of starch granules are directly related to the branch chain length of amylopectin. ${ }^{2)}$ The A-type starch consists of more short branch chains, and the B-type starch consists of more long B chains. $^{32,42)}$ The A-type starch granules also show pinholes on the surface and serpentine-like channels inside of the granule, but the B-type starch granules do not show these features. ${ }^{56,57)}$ Using a gas absorption method, Hellman and Melvin find that maize starch granules consist of more total surface area than the surface area of the starch granules, whereas the total surface area of potato starch 
granules is similar to the surface area of the starch granules. $^{58)}$

Recent studies using confocal laser-scattering microscopy (CLSM) have shown that sorghum and maize starch, when stained with methanolic merbromin ${ }^{57)}$ or derivatized with ionic analog of propylene oxide and silver nitrate and detection of silver metal, ${ }^{59)}$ display a large number of channels. The pinhole structure has also been reported by Gallant and other researchers. ${ }^{47)}$ Using rhodamine Bstained starch and having excess dye removed, the A-type starch granules (e.g., sugary-2, waxy maize and normal maize starch) show substantial amount of voids (Figs. 6A, $\mathrm{B}$ and $\mathrm{C}$, respectively), ${ }^{60)}$ which are likely the results of loosely packed starch molecules that do not bind the dye tightly. The loosely packed structure of the A-type polymorphic starch could be attributed to that the A-type polymorphic starch granules consist of large populations of short branch-chains (A and B1 chains). The short A and B1 chains in the crystalline structure are less stable and are more susceptible for rearrangement and generate more loosely packed areas of voids. The B-type polymorphic (potato and high-amylose maize) and some C-type polymorphic (banana) starch have more long B chains, which extend through two or more clusters and stabilize the internal structures of granules (Figs. 6D, E and F, respectively).

The CLSM images of the internal structures of the starch granules are in agreement with the scanning electron micrographs of the remaining starch granules after the peripheral starch has been removed by surface gelatinization of starch granules (Fig. 7). ${ }^{24,25)}$ After the starch granules have been treated with a saturated neutral saltsolution (e.g., $\mathrm{CaCl}_{2}$ and $\mathrm{LiCl}$ ) for a selected time period and the surface starch has been gelatinized and removed, the remaining granules of the potato starch display a solid structure (Fig. 7A). In contrast, the remaining granules of the normal maize starch display fissures, opens and loosely packed structures (Fig. 7B). This loosely packed starch structure of maize starch granules, in which amylose molecules are not firmly embedded among amylopectin crystallites, can be responsible for some of its characteristic properties, including amylose leaching, iodine vapor complexing with amylose to give blue color and DMSO solubilization of maize starch to cause splitting of starch granules. ${ }^{14)}$

Starch granules isolated from corn kernels harvested at different maturation stages, 16, 20, 30 DAP and mature/ dried kernels (45 DAP) have been investigated using scanning electronmicroscopy. ${ }^{27)}$ Starch granules isolated from the mature/dried kernels (45 DAP) show a substantial number of pinholes. Starch granules isolated from kernels of 30 DAP show few pinholes, whereas starch granules from kernels of 20 DAP and earlier do not show pinholes (Fig. 8). ${ }^{27)}$ These results suggest that the pinholes are likely produced by amylase attack and hydrolysis of starch granules on the surface during the maturation and drying of corn in the field. Mature corn kernels, even at the dormant state, can produce amylases, possibly induced by signals of germination. The indigenous amylase can hydrolyze starch from the surface and produce pinholes and channels.
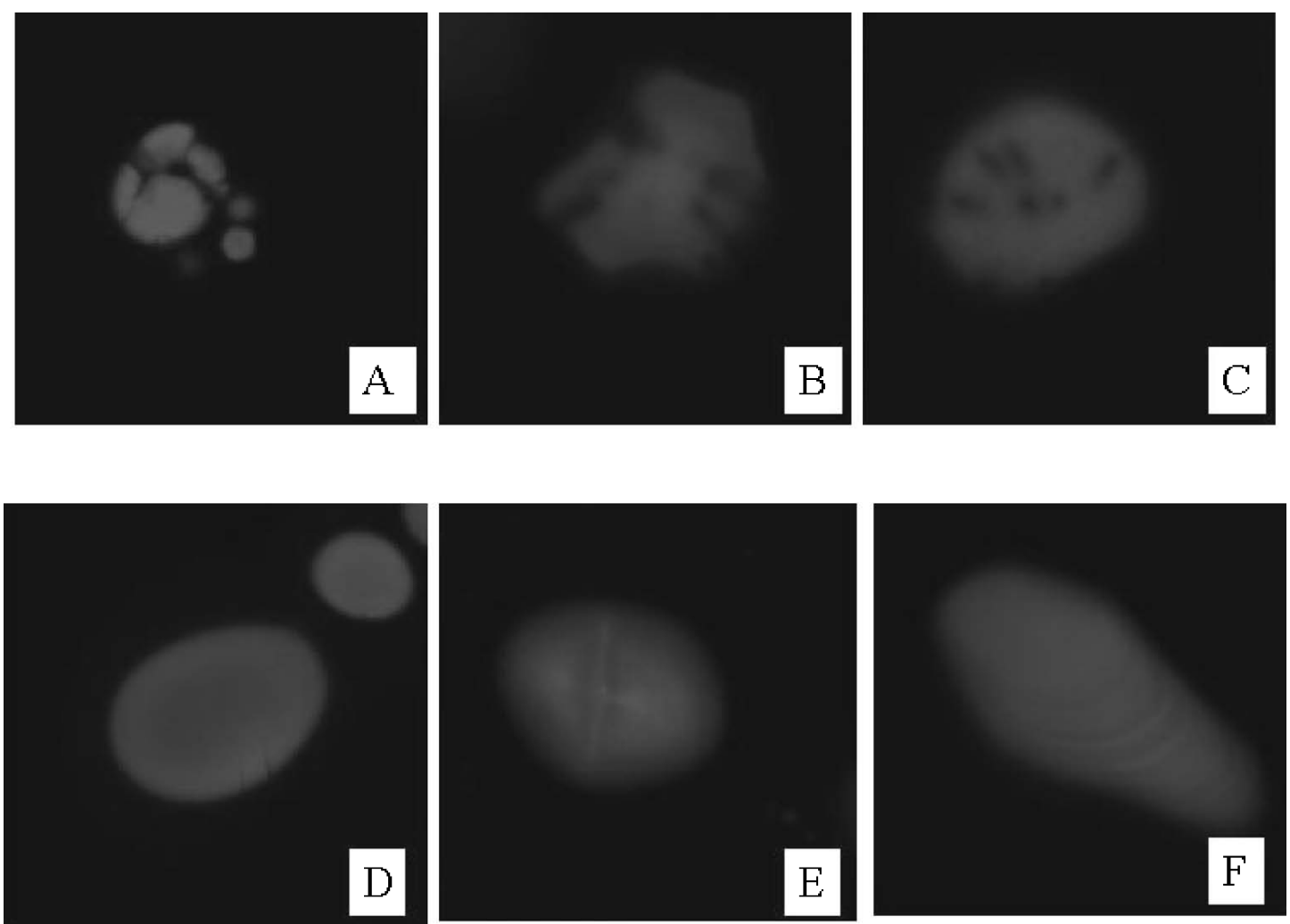

Fig. 6. Confocal laser-light scanning micrographs of starch granules.

Starch was stained with rhodamine B, and unbound dye was removed by rinsing with water and centrifuged immediately. (A) Sugary-2 maize starch, (B) waxy maize starch, (C) normal maize starch, (D) potato starch, (E) high-amylose maize VII starch and (F) banana starch. Reprinted with permission from Ref. 60). 
A

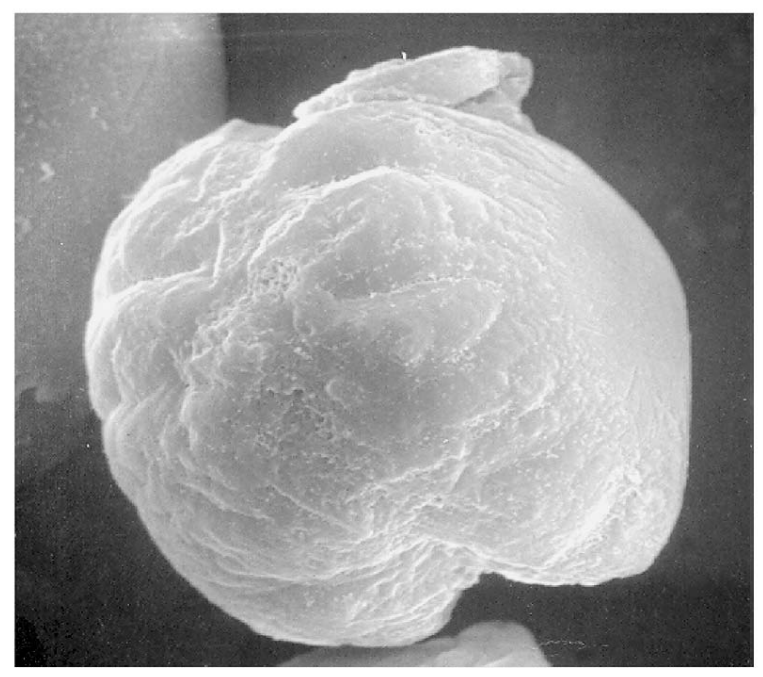

B

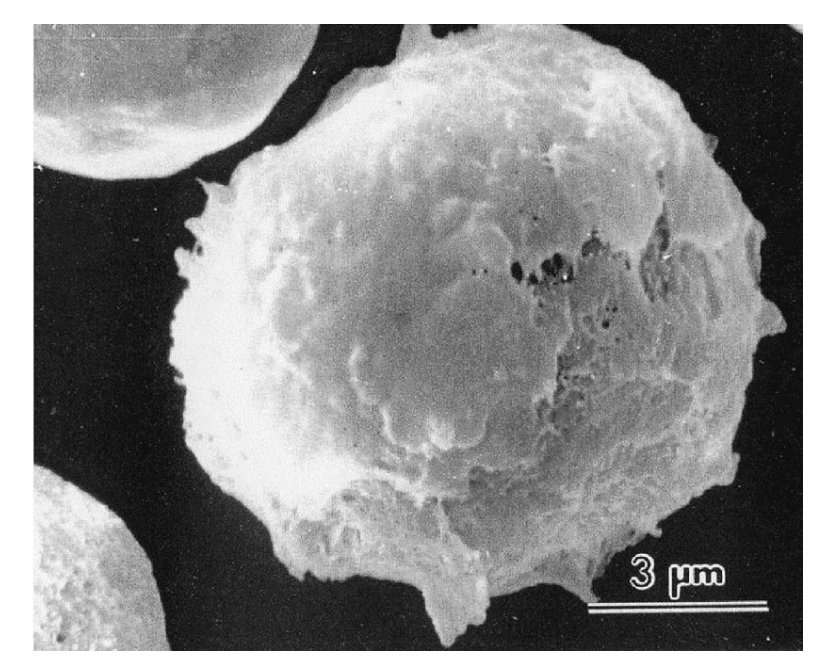

Fig. 7. Surface gelatinized remaining starch granules.

(A) Potato starch after being treated with a saturated $\mathrm{CaCl}_{2}$ solution and $\sim 65 \%$ surface starch removed remaining granule, (B) normal maize starch after being treated with a saturated $\mathrm{LiCl}$ solution and $65 \%$ surface starch removed remaining granule.

\section{Summary.}

Amylose is interspersed among amylopectin and is more concentrated at the periphery than at the core of a starch granule. Amylopectin at the periphery of a starch granule consisits of shorter branch chains than that at the inner part of the granule. Amylopectin of the disk-shaped A-granule of wheat, triticale and barley starch consists of more B2 chains and fewer short A and B1 chains, which has a cylindrical shaped molecule and better fits to the disk shaped granule. Amylopectin of the spherical Bgranule has more short $\mathrm{A}$ and $\mathrm{B} 1$ chains and fewer B2 chains, which has a cone-shaped molecules. The A-type polymorphic starch granules have loosely packed internal structures, which are easily hydrolyzed by amylases, whereas the B-type polymorphic starch granules have solid internal structures and are resistant to enzyme hydrolysis.
A

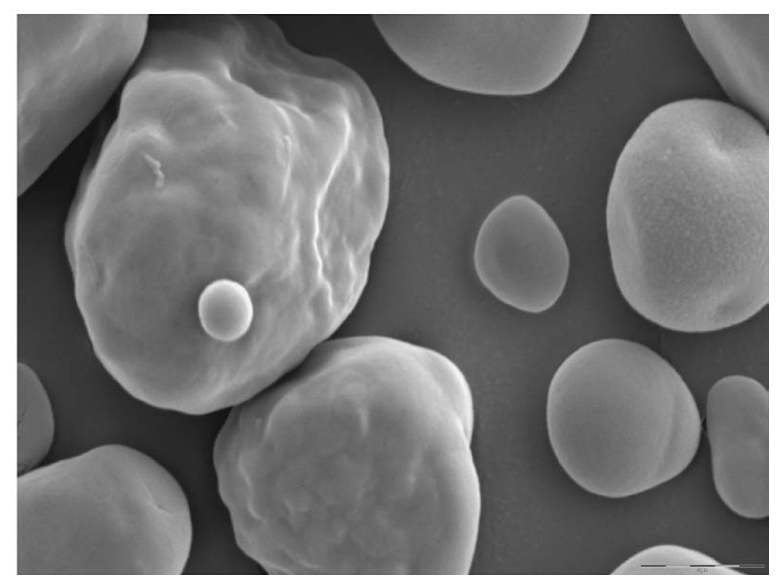

B

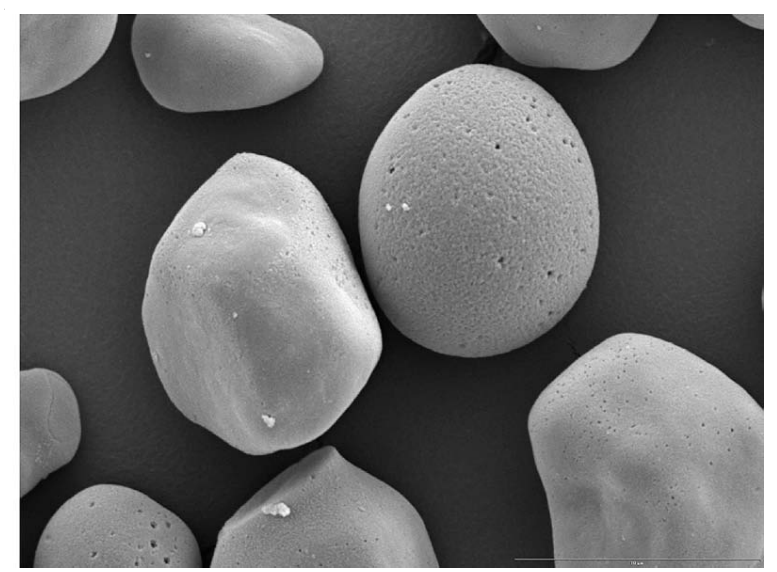

Fig. 8. Scanning electron micrographs of normal maize (B73) starch granules.

(A) Starch granules isolated from kernels harvested on 30 days after pollination (DAP); little or no pinholes are observed, (B) the starch isolated from kernels harvested on 45 DAP, which are mature and dried, show many pinholes on the surface of the granules.

The author thanks Dr. Keiji Kainuma for reviewing the manuscript and providing invaluable discussion and suggestions.

\section{REFERENCES}

1 ) J. Jane, T. Kasemsuwan, S. Leas, H. Zobel and J.F. Robyt: Anthology of starch granule morphology by scanning electron microscopy. Starch/Stärke, 46, 121-129 (1994).

2 ) S. Hizukuri: Relationship between the distribution of the chain length of amylopectin and the crystalline structure of starch granules. Carbohydr. Res., 141, 295-306 (1985).

3 ) A. Imberty, A. Buleon, V. Tran and S. Perez: Recent advances in knowledge of starch structure. Starch/Stärke, 43, 375-384 (1991).

4 ) H.F. Zobel: Starch crystal transformations and their industrial importance. Starch/Stärke, 40, 1-7 (1988).

5 ) H. Fuwa, T. Takaya and Y. Sugimoto: Degradation of various starch granules by amylases. in Mechanisms of Saccharide of Polymerization and Depolymerization, J.J. Marshall, ed., Academic Press, New York, NY, pp. 73-102 (1980).

6 ) A. Kimura and J.F. Robyt: Reaction of enzyme with starch granules: kinetics and products of the reaction with glucoamylase. Carbohydr. Res., 277, 87-107 (1995).

7 ) K.E. Spence and J. Jane: Chemical and physical properties of ginkgo (GINKGO GILOBA) starch. Carbohydr. Polym., 40, 
261-269 (1999).

8 ) C. Perera, Z. Lu, J. Sell and J. Jane: Comparison of physicochemical properties and structures of sugary-2 cornstarch with normal and waxy cultivars. Cereal Chem., 78, 249-256 (2001).

9 ) J. Jane, Z. Ao, S.A. Duvick, S.-H. Yoo, K.-S. Wong and C. Gardner: Structures of amylopectin and starch granules: How are they synthesized? J. Appl. Glycosci., 50, 167-171 (2003).

10) S. Hizukuri, Y. Takeda and M. Yasuda: Multi-branched nature of amylose and the action of debranching enzymes. Carbohydr. Res., 94, 205-213 (1981).

11) Y. Takeda, K. Shirasaka and S. Hizukuri: Examination of the purity and structure of amylose by gel-permeation chromatography. Carbohydr. Res., 132, 83-92 (1984).

12) Y. Takeda and S. Hizukuri: Structure of branched molecules of amyloses of various origins, and molar fractions of branched and unbranched molecules. Carbohydr. Res., 165, 139-145 (1987).

13) J. Jane and J.F. Chen: Effect of amylose molecular size and amylopectin branch chain length on paste properties of starch. Cereal Chem., 69, 60-65 (1992).

14) H.F. Zobel: Molecules to granules: A comprehensive starch review. Starch/Stärke, 44, 44-50 (1988).

15) J. Jane, S.A.S. Craig, P.A. Seib and R.C. Hoseney: Characterization of granular cold water-soluble starch. Starch/Stärke, 38, 258-263 (1986).

16) J. Jane, A. Xu, M. Rodosovljivic and P.A. Seib: Location of amylose in normal starch granules. I. Susceptibility of amylose and amylopectin to cross-linking reagents. Cereal Chem., 69, 405-409 (1992).

17) T. Kasemsuwan and J. Jane: Location of amylose in normal starch granules. II. Locations of phosphor-diesters and -monoesters revealed by ${ }^{31} \mathrm{P}-\mathrm{nmm}$. Cereal Chem., 71, 282-287 (1994).

18) M. Yoshida, M. Fujii, Z. Nikuni and B. Maruo: The appositive growth of starch granules in beans as revealed by autoradiography. Bull. Agric. Chem. Soc. Jpn., 22, 127 (1958).

19) T. Baba, M. Yoshii and K. Kainuma: Incorporation of glucose to the outer chain of starch granule. Starch/Stärke, 39, 53-56 (1987).

20) J. Preiss and M. Sivak: Starch synthesis in sinks and sources. in Photoassimilate Distribution in Plants and Crops, E. Zamski and A.A. Schaffer, eds., Marcel Dekker, New York, pp. 63-96 (1996).

21) Y. Nakamura: Towards a better understanding of the metabolic system for amylopectin biosynthesis in plants: rice endosperm as a model tissue. Plant Cell Physiol., 43, 718-725 (2002).

22) K. Denyer, B. Clarke, C. Hylton, H. Tatge and A.M. Smith: The elongation of amylose and amylopectin chains in isolated starch granules. Plant J., 10, 1135-1143 (1996).

23) W.R. Morrison and H.J. Gadan: The amylose and lipid contents of starch granules in developing wheat endosperm. $J$. Cereal Sci., 5, 263-275 (1987).

24) J. Jane and J.J. Shen: Internal structure of the potato starch granule revealed by chemical gelatinization. Carbohydr. Res., 247, 279-290 (1993).

25) D.D. Pan and J. Jane: Internal structure of normal maize starch granules revealed by chemical surface gelatinization. Biomacromolecules, 1, 126-132 (2000).

26) H. Yoshida, K. Nozaki, I. Hanashiro, F. Yagi, H. Ito, M. Honma, H. Matsui and Y. Takeda: Structure and physicochemical properties of starches from kidney bean seeds at immature, premature and mature stages of development. Carbohydr. Res., 338, 463-469 (2003).

27) L. Li: Starch biogenesis: relationship between starch structures and starch biosynthetic enzymes. Ph.D. Dissertation. Iowa State University, Ames, IA, USA (2006).

28) I. Dry, A. Smith, A. Edwards, M. Bhattacharyya, P. Dunn and C. Martin: Characterization of cDNAs encoding two isoforms of granule-bound starch synthase which show differential expression in developing storage organs of pea and potato. Plant J., 2, 193-202 (1992).

29) P.L. Vrinten and T. Nakamura: Wheat granule-bound starch synthase I and II are encoded by separate genes that are ex- pressed in different tissues. Plant Physiol., 122, 255-264 (2000).

30) S. Yoo and J. Jane: Structural and physical characteristics of waxy and other wheat starches. Carbohydr. Polym., 49, 297305 (2002).

31) N. Inouchi, H. Hibiu, T. Li, T. Horibata, H. Fuwa and T. Itani: Structure and properties of endosperm starch from cultivated rice from Asia and other countries. J. Appl. Glycosci., 52, 239-246 (2005).

32) J. Jane, Y.Y. Chen, L.F. Lee, A.E. McPherson, K.S. Wong, M. Radosavljevic and T. Kasemsuwan: Effects of amylopectin branch chain length and amylose content on the gelatinization and paste properties of starch. Cereal Chem., 76, 629-637 (1999).

33) M.L. Fishman, L. Rodriguez and H.K. Chau: Molar masses and sizes of starches by high-performance size exclusion chromatography with on-line multiangle laser light scattering detection. J. Agric. Food Chem., 44, 3182-3188 (1996).

34) S. Yoo and J. Jane: Molecular weights and gyration radii of amylopectins determined by high-performance size-exclusion chromatography equipped with multi-angle laser light scattering and refractive index detection. Carbohydr. Polym., 49, 307-314 (2002).

35) K.S. Wong and J. Jane: Quantitative analysis of debranched amylopectin by HPAEC-PAD with a postcolumn enzyme reactor. J. Liquid. Chromatogr., 20, 297-310 (1997).

36) M. Gao, D.K. Fisher, K. Kim, J.C. Shannon and M.J. Guiltinan: Evolutionary conservation and expression patterns of maize starch branching enzyme I and IIb genes suggests isoforms specialization. Plant Mol. Biol., 30, 1223-1232 (1996).

37) Z. Nikuni: Studies on starch granules. Staerke/Starch, 30, 105 $-111(1978)$

38) D. French: Fine structure of starch and its relation to the organization of starch granules. Denpun Kagaku, 19, 8-25 (1972).

39) J.P. Robin, C. Mercier, R. Charbonniere and A. Guilbot: Lintnerized starches. Gel filtration and enzymatic studies of insoluble residues from prolonged acid treatment of potato starch. Cereal Chem., 51, 389-406 (1974).

40) M. Yamaguchi, K. Kainuma and D. French: Electron microscopic observations of waxy maize starch. J. Ultrastruct. Res., 69, 249-261 (1979).

41) D.R. Lineback: The starch granule organization and properties. Bakers Digest, 16-21 (1984).

42) S. Hizukuri: Polymodal distribution of the chain lengths of amylopectins and its significance. Carbohydr. Res., 147, 342347 (1986).

43) M.J. Gidley and P.V. Bulpin: Crystallisation of maltooligosaccharides as models of the crystalline forms of starch: minimum chain-length requirement for the formation of double helices. Carbohydr. Res., 161, 291-300 (1987).

44) S.G. Ring, M.J. Miles, V.J. Morris and R. Turner: Spherulitic crystallization of short chain amylose. Int. J. Biol. Macromol., 9, 158-160 (1987).

45) G.T. Oostergetel and E.F.J. ven Bruggen: The crystalline domains in potato starch are arranged in a helical fashion. Carbohydr. Polym., 21, 7-12 (1993).

46) E. Bertoft and K. Koch: Composition of chains in waxy-rice starch and its structural units. Carbohydr. Polym., 41, 121-132 (2000).

47) D.J. Gallant, B. Bouchet and P.M. Baldwin: Microscopy of starch: evidence of a new level of granule organization. Carbohydr. Polym., 32, 177-191 (1997).

48) K. Kainuma and D. French: Naegeli amylodextrin and its relationship to starch granule structure. II Role of water in crystallization of B-starch. Biopolymers, 11, 2241-2250 (1972).

49) K. Umeki and K. Kainuma: Fine structure of Naegeli amylodextrin obtained by acid treatment of defatted waxy-maize starch-structural evidence to support the double-helix hypothesis. Carbohydr. Res., 96, 143-159 (1981).

50) J. Jane, K.-S. Wong and A.E. McPherson: Branch-structure difference in starches of A- and B-type x-ray patterns revealed by their Naegeli dextrins. Carbohydr. Res., 300, 219-227 (1997). 
51) P.J. Jenkins, R.E. Cameron and A.M. Donald: A universal feature in the structure of starch granules from different botanical sources. Starch/Stärke, 45, 417-420 (1993).

52) L.F. Hood and C. Mercier: Molecular structure of unmodified and chemically modified manioc starches. Carbohydr. Res., 61, 53-66 (1978).

53) Y. Takeda, C. Takeda, H. Mizukami and I. Hanashiro: Structures of large, medium and small starch granules of barley grain. Carbohydr. Polym., 38, 109-114 (1999).

54) Z. Ao and J. Jane: Characterization of A- and B-granule starches of barley, wheat, and triticale. Carbohydr. Polym., in press (2006).

55) H. Tang, K. Watanabe and T. Mitsunaga: Structure and functionality of large, medium and small granule starches in normal and waxy barley endosperms. Carbohydr. Polym., 49, 217224 (2002)

56) J.E. Fannon, R.J. Huber and J.N. BeMiller: Surface pores of starch granules. Cereal Chem., 69, 284-288 (1992).

57) K.C. Huber and J.N. BeMiller: Channels of maize and sorghum starch granules. Carbohydr. Polym., 41, 269-276 (2000).

58) N.N. Hellman and E.H. Melvin: Surface area of starch and its role in water sorption. J. Am. Chem. Soc., 72, 5186-5188 (1950).

59) J.A. Gray and J.N. BeMiller: Development and utilization of reflectance confocal laser scanning microscopy to locate reaction sites in modified starch granules. Cereal Chem., 81, 278286 (2004).

60) J. Jane, N. Atichokudomchai and D.-S. Suh: Internal structures of starch granules revealed by confocal laser-light scanning microscopy. in Starch: Progress in Structural Studies, Modifications and Applications, P. Tomasik, V.P. Yuryev and E. Bertoft, eds., Polish Society of Food Technologists, Cracow, pp. 147-156 (2004). 